
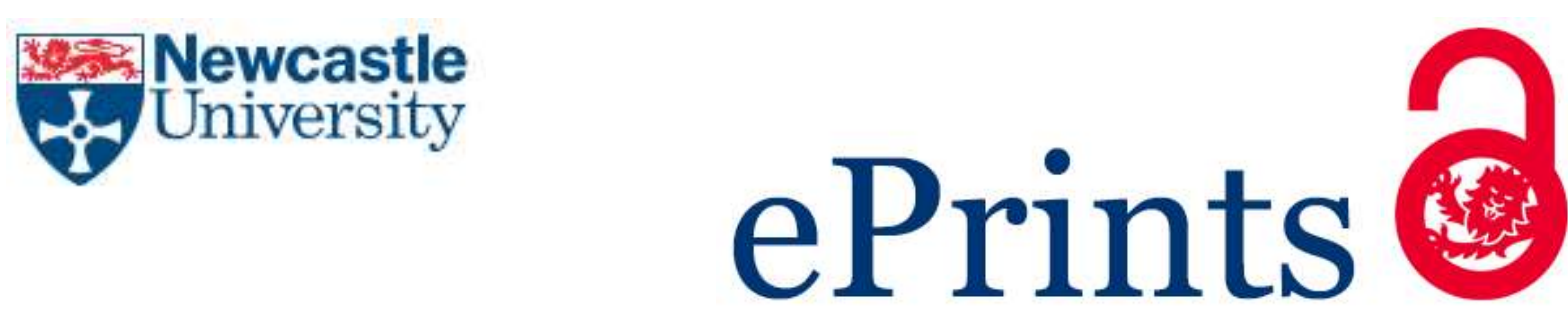

Martin TJ, Snape JR, Bartram A, Robson A, Acharya K, Davenport RJ. Environmentally relevant inoculum concentrations improve the reliability of persistent assessments in biodegradation screening tests. Environmental Science and Technology 2017, 51(5), 3065-3073.

\title{
Copyright:
}

(C) ACS Author Choice - This is an open access article published under a Creative Commons Attribution (CC-BY) License, which permits unrestricted use, distribution and reproduction in any medium, provided the author and source are cited.

DOI link to article:

http://dx.doi.org/10.1021/acs.est.6b05717

Date deposited:

$30 / 03 / 2017$

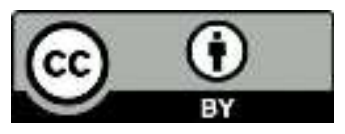

This work is licensed under a Creative Commons Attribution 4.0 International License 


\section{Environmentally Relevant Inoculum Concentrations Improve the Reliability of Persistent Assessments in Biodegradation Screening Tests}

Timothy J. Martin, ${ }^{*}{ }^{\dagger}$ ○ Jason R. Snape, ${ }^{\ddagger}$ Abigail Bartram, ${ }^{\ddagger}, \S$ Aidan Robson, ${ }^{\dagger}$ Kishor Acharya, ${ }^{\dagger}$ and Russell J. Davenport ${ }^{\dagger}$

${ }^{\dagger}$ School of Civil Engineering and Geosciences, Cassie Building, Newcastle University, Newcastle upon Tyne, NE1 7RU, United Kingdom

${ }^{\ddagger}$ AstraZeneca Global Environment, Mereside, Alderley Park, Macclesfield, Cheshire, SK10 4TG, United Kingdom

Supporting Information

ABSTRACT: Standard OECD biodegradation screening tests (BSTs) have not evolved at the same rate as regulatory concerns, which now place an increased emphasis on environmental persistence. Consequently, many chemicals are falsely assigned as being potentially persistent based on results from BSTs. The present study increased test duration and increased inoculum concentrations to more environmentally relevant levels to assess their impact on biodegradation outcome and intratest replicate variability for chemicals with known environmental persistence. Chemicals were assigned to potential persistence categories based on existing degradation data. These more environmentally relevant BSTs (erBSTs) improved the reliability of persistence assignment by reducing the high variability associated with these tests and the occurrence of failures at low inoculum concentrations due to the exclusion of specific degraders. Environmental fate was determined using a reference set of ${ }^{14} \mathrm{C}$-labeled compounds with a range of potential environmental persistences, and full mass balance data were collated. The erBST correctly assigned five reference chemicals of known biodegradabilities to their appropriate persistence category in contrast to a standard OECD Ready Biodegradation Test (RBTs, $P<0.05$ ). The erBST was significantly more reproducible than an OECD RBT (ANOVA, $P<0.05$ ), with more consistent rates and extent of biodegradation observed in the erBST.

\section{INTRODUCTION}

Regulatory frameworks (e.g., registration, evaluation, authorization, and restriction of chemicals (REACH), Biocidal Products Directive (BPD), European Directives on medicinal products for human use, and Veterinary Medicines Directive (VMD)) coupled with supporting technical guidance and standardized test guidelines help to protect the environment and human health from the risks and hazards posed by hundreds of thousands of globally manufactured chemicals. Tests for biodegradation are an important part of these regulatory frameworks as they help to assess the likelihood that a chemical will persist in the environment and increase its potential for environmental exposure. In recent years, biodegradation screening tests (BSTs), which includes the OECD Ready Biodegradability Test series ${ }^{1}$ and inherent biodegradability tests, have not evolved at the same rate as these regulatory frameworks and emerging environmental concerns. ${ }^{2}$ A much greater emphasis is now placed on identifying and prioritizing chemicals based on their environmental hazardous properties: persistence, bioaccumulation, and toxicity (PBT), rather than their environmental risk alone. Environmental persistence is defined by a series of environmental half-life thresholds, e.g., $>40$ days for freshwater. ${ }^{3}$ Ready biodegradability tests (RBTs) remain as a first tier screening test in environmental exposure and persistence assessment; ${ }^{2}$ a pass is required to identify chemicals that are unlikely to persist in the environment and undergo rapid ultimate biodegradation. RBTs are stringent tests which were originally introduced into regulatory testing over 30 years ago to provide an assessment of chemical fate and in doing so screen out chemicals which would rapidly degrade in all environments during their routine use. ${ }^{1}$ They have historically formed the foundation of all biodegradation assessments for hazard identification (for classification and labeling), environmental risk assessment, and now persistence assessment. This has been largely due to their relatively low cost, perceived standardization, and straightforward implementation and interpretation. OECD RBTs are pass/fail tests focusing on

Received: November 12, 2016

Revised: January 4, 2017

Accepted: January 26, 2017

Published: January 26, 2017 
mineralization of the test chemical or dissolved organic carbon (DOC) removal, with the chemical introduced at high concentrations as a sole carbon source in the presence of a diluted bacterial inoculum sourced from the environment under defined laboratory conditions over 28 days. ${ }^{1}$ They are deemed conservative as they are designed to detect only growth-linked degradation using inocula that are four to five orders of magnitude lower in concentration than those typically found in the natural environment. This dilution in the inoculum concentration also reduces the microbial diversity introduced into the test and can exclude less abundant members of the community being sampled. ${ }^{4,5}$ There are seven RBT formats, six that comprise the OECD 301 series and the OECD 310 test. ${ }^{1}$

RBTs suffer a number of well-documented limitations (see Kowalczyk et $\mathrm{al}^{2}$ for a more extensive review) that are particularly pertinent to their use in persistence assessment. These limitations are (i) the high number of test fails, (ii) the high variability between replicates and repeat studies, (iii) the ability to only detect growth-linked kinetics where the test compound is the carbon source, and (iv) the arbitrary time restriction (namely, a 28 day duration in which the pass criterion is reached 10 days after $10 \%$ degradation has been achieved). The first and second of these limitations can be attributed to the variation in test formats and the low inocula concentrations used in the tests. The origins and scientific evidence for the arbitrary time restrictions are unknown, ${ }^{6}$ although the test duration falls short of the half-life threshold for classifying persistence. Persistence assessments are focused on chemicals likely to be widely distributed in the environment at relatively low concentrations; therefore, first order kinetics are of greatest relevance. ${ }^{7}$ RBTs were designed to screen chemicals at high concentrations (typically $10-100 \mathrm{mg} / \mathrm{L}$ ). They therefore typically exhibit mixed order kinetics depending on the substrate concentration and the substrate affinity of the population: zero order when the chemical concentration is high enough for biodegradation to be independent of concentration and first order at lower chemical concentrations, though little of this fundamental knowledge is considered within the current tests or their interpretation. Notwithstanding these limitations, the high number of test fails and high variability are perhaps the most important issues from a chemical hazard and risk assessment perspective.

High Number of Test Fails. It has been estimated that there is a $20-80 \%$ chance that a biodegradable chemical can be falsely classified as potentially persistent in current RBTs, i.e., a false negative. ${ }^{8}$ Under the test criteria, a chemical can be designated as not "readily biodegradable" if one replicate fails to reach the arbitrarily designated pass criteria (60\% or $70 \%$ degradation depending on the end-point being analyzed). In persistence assessments, these chemicals would have to undergo potentially unnecessary and costlier higher-tier biodegradation tests, which can be difficult to interpret, ${ }^{9}$ and/or bioaccumulation and toxicity tests (depending on the $\log K_{\mathrm{ow}}$ of the chemical), with the implicit animal welfare issue it raises.

High Variability. RBTs have shown high variability in the extent of biodegradation and/or lag phase between different tests, ${ }^{10-13}$ within the same facility or between different facilities, ${ }^{6,14,15}$ between the same test using different analytical methods, ${ }^{16}$ and within test replicates ${ }^{17,18}$ for the same chemical. Such variation may reflect differences in the analytical methods used with different RBTs (oxygen demand, carbon dioxide evolution, and dissolved organic carbon removal) and their interpretation. However, most studies show that low inoculum concentration, small test volumes, and lack of adaptation of the inoculum in the presence of the chemical prior to the test, have a significant influence on (i) the observed variation, (ii) the final outcome of an RBT, and (iii) the duration of the lag phase such that they generate false negatives. ${ }^{18-21}$ These studies infer that there is an increased chance of excluding specific degraders of nonpersistent chemicals into the test by capturing only a small proportion of the bacteria that would be encountered in the environment and/or not allowing them sufficient time to adapt (thereby resulting in long lag phases and test fails). They suggest that the reliability and relevance of RBTs would be improved by increasing the inoculum concentration, ${ }^{17,18}$ increasing the test volume, ${ }^{22}$ or including an adaptation phase into the test. ${ }^{23-25}$ Indeed, the OECD RBT guidance also acknowledges that a reduction in variation between replicates can be achieved with increasing inoculum concentration. ${ }^{1}$ Based on the above evidence, a number of enhancements and modifications to existing OECD BSTs have been identified to enable more effective prioritization of persistence. These include increasing the total number of cells in a test by increasing inoculum concentrations, increasing the volume of tests and extending test duration to capture adaptation and growth-linked biodegradation to encompass the half-life persistence threshold duration. ${ }^{4,8,26}$ These also form part of the current REACH guidelines $^{27}$ due for a second round of drafting in 2016.

In this study, we compared a conventional RBT, based on the OECD 301B, ${ }^{1}$ with an equivalent method using more environmentally relevant inocula concentrations. The ability of these tests to accurately differentiate persistent chemicals from nonpersistent ones was validated with respect to regulatory criteria for ready biodegradability and persistence using radiolabeled reference chemicals. Reference chemicals with known biodegradability characteristics, based on their current ECHA Brief Profile classifications on biodegradation ${ }^{28}$ and the availability of extensive degradation data for these chemicals, ${ }^{29}$ were selected for this validation exercise.

\section{EXPERIMENTAL SECTION}

These studies were conducted in compliance to Good Laboratory Practice (GLP) in an accredited laboratory.

Sampling and Inoculum Preparation. Activated sludge (AS) was obtained from Buckland Sewage Treatment Works, Devon, U.K., which has a population equivalence of approximately 80000 and treats predominantly domestic sewage. AS was sampled on three occasions to provide inocula for three studies, the data from which is collated in this manuscript. AS was not pretreated, other than amendment with sterile OECD mineral media, ${ }^{1}$ to give total cell counts determined using epifluorescence microscopy following staining with $4^{\prime}, 6-$ diamidino-2-phenylindole (DAPI, Sigma-Aldrich, U.K.) of $10^{5}-10^{7}$ cells $\mathrm{mL}^{-1},{ }^{30}$ the upper range of which are more representative of total cell concentrations in activated sludge wastewater treatment systems. ${ }^{18}$

Test Chemicals. Five test chemicals were selected with a range of environmental persistence based on extensive half-life and test data previously reviewed for this purpose $^{29}$ (Table 1). Three of the chemicals selected are known to give rise to variable persistence outcomes in standard RBTs. Chemicals were assigned to the following persistence categories based on their current ECHA Brief Profile classifications on biodegradation ${ }^{28}$ (Table 1); nonpersistent and readily biodegradable (aniline, ANI; 4-fluorophenol, 4-FP); nonpersistent and inherently or variably biodegradable (4-nitrophenol, 4-NP; 4-chloroaniline, 


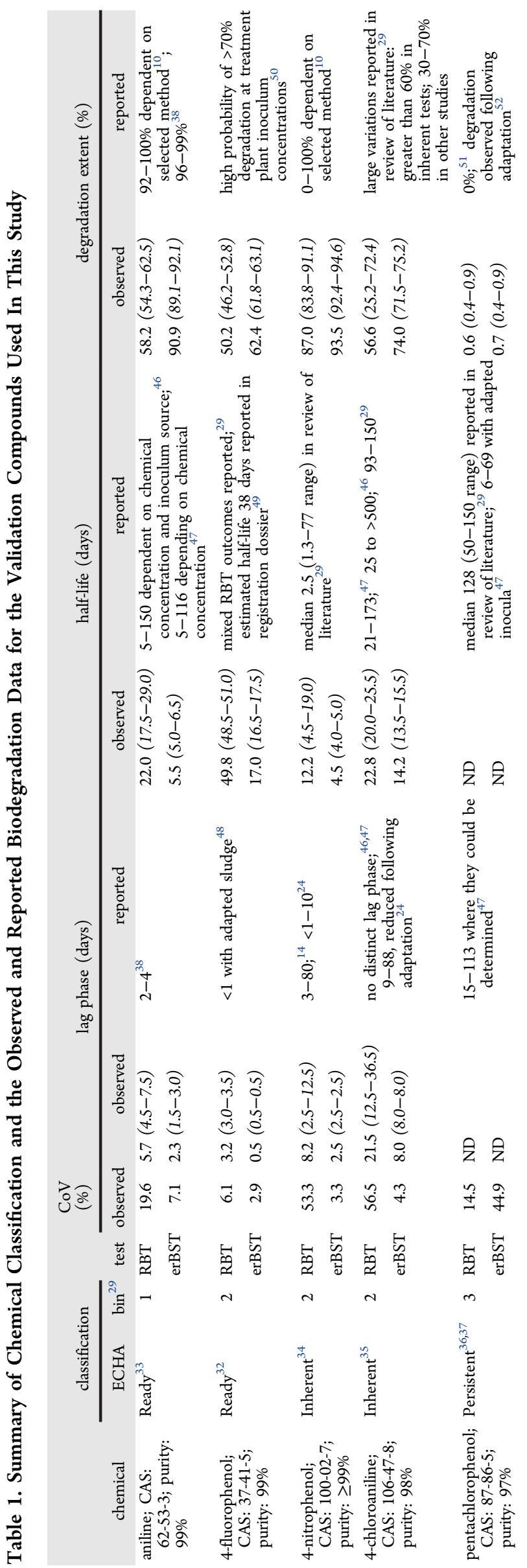

4-CA); or persistent (pentachlorophenol, PCP). Chemical purity ranged from $97 \%$ to $\geq 99 \%$ (Table 1). Concentrated stock solutions $\left(1 \mathrm{~g} \mathrm{C} \mathrm{L} \mathrm{L}^{-1}\right)$ of reference chemicals (SigmaAldrich, Poole, U.K.) were prepared in OECD mineral media. ${ }^{1}$ Concentrated stock solutions were combined with radiolabeled stock solutions of the test chemicals (universally labeled ${ }^{14}$ C: ARC Inc., St. Louis, MO, U.S.A.), and radioactivity was determined via liquid scintillation counting (LSC) (Tri-Carb 2800-TR, PerkinElmer, Waltham, MA, USA). Tests were dosed at a ratio of $10 \mathrm{~mL}$ of dosing stock to $1 \mathrm{~L}$ of test inoculum, to give final test concentrations of $10 \mathrm{mg} \mathrm{C} \mathrm{L}^{-1}$. The level of radioactivity applied allowed less than $1 \%$ degradation to be resolved in the sampled $\mathrm{NaOH}$, with an average dose of $27 \mathrm{~Bq} \mathrm{~mL}{ }^{-1}$ (ranging from 17 to $59 \mathrm{~Bq} \mathrm{~mL}^{-1}$ ).

Biodegradation Screening Test (BST) Preparation. Two sets of BSTs were performed, one set as per OECD $301 \mathrm{~B}$ test guidelines, ${ }^{1}$ using $3 \mathrm{mg} \mathrm{L}^{-1}$ of inoculum (a typical OECD RBT concentration, in the order of $10^{5}$ cells $\mathrm{mL}^{-1}$ ) and another set, which were essentially the same but with inocula 100 times more concentrated at $300 \mathrm{mg} \mathrm{L}^{-1}$. This inocula provided more environmentally relevant concentrations but was still an order of magnitude lower than those used in tests for inherent biodegradability (OECD 302), except the modified MITI test (OECD 302C). Those tests with $300 \mathrm{mg} \mathrm{L}^{-1}$ were termed environmentally relevant BSTs (erBSTs), which have been deemed "enhanced" tests in technical guidance documents. ${ }^{3,8}$ Each test was performed in triplicate. Air was drawn through an influent $\mathrm{CO}_{2}$ scrubber $(50 \mathrm{~mL}$ of $2 \mathrm{M} \mathrm{NaOH})$ and humidifier $\left(50 \mathrm{~mL}\right.$ of $\left.\mathrm{H}_{2} \mathrm{O}\right)$ prior to the test vessel incorporating test inocula $(977 \mathrm{~mL})$, OECD mineral media $(10 \mathrm{~mL}$ of solution A, $1 \mathrm{~mL}$ each of solutions $\mathrm{B}, \mathrm{C}$, and $\mathrm{D}){ }^{1}{ }^{1}$ and test chemical $(10 \mathrm{~mL})$. Evolved ${ }^{14} \mathrm{CO}_{2}$ was captured in traps containing $50 \mathrm{~mL}$ of $2 \mathrm{M} \mathrm{NaOH}$, positioned after the main test vessel with empty traps positioned on either side of the $\mathrm{NaOH}$ traps, and quantified as a measure of ultimate biodegradation by liquid scintillation counting (LSC). Orbo tubes (Orbo 32 and 91, Sigma-Aldrich, Poole, U.K.) were incorporated between the test vessel and the $\mathrm{NaOH}$ traps to capture volatilized test compounds and degradation products. Test systems were prepared in triplicate for each chemical and inoculum concentration. Test systems were maintained aerobic at $20^{\circ} \mathrm{C}\left( \pm 2{ }^{\circ} \mathrm{C}\right)$ in the dark and run for 60 days.

Biodegradation Determination and Interpretation. During periodic sampling, ${ }^{14} \mathrm{CO}_{2}$ captured in $2 \mathrm{M} \mathrm{NaOH}$ traps was collected in preweighed $1 \mathrm{~L}$ Nalgene bottles (Thermo-Fisher Scientific, Waltham, MA, U.S.A.), which were subsequently reweighed. Triplicate $5 \mathrm{~mL} \mathrm{NaOH}$ subsamples were mixed with a Gold Star scintillation cocktail (Meridian Biotechnologies Ltd., U.K.) and analyzed via LSC. The accumulated radioactivity was converted to a percentage of the originally applied radioactivity and used as an indicator of ultimate biodegradation.

Biodegradation outcome was assessed based both on OECD $\mathrm{RBT}^{1}$ pass thresholds and recommendations for persistence assessments. ${ }^{27}$ For ready biodegradability, the OECD RBT pass threshold is $60 \%$ degradation within 28 days, and additionally the pass threshold must be reached within a 10-day window beginning once degradation has reached $10 \%$. Chemicals exhibiting half-lives greater than 40 days in freshwater were classified as persistent based on recommendations for persistence assessments ${ }^{27}$ over the 28 and 60 day period for both tests. In the current testing regime, a chemical found to readily biodegrade is classified as nonpersistent (not $\mathrm{P}$ ). Test chemicals 
failing to achieve the pass threshold (60 or $70 \%$ depending on the end point) within 28 days are deemed potentially persistent and may need to undergo additional testing. ${ }^{27}$

Biodegradation descriptors were used to make comparisons between replicates and between the RBT and the erBST. These descriptors included lag phase $\left(t_{\mathrm{L}}\right)$, the time taken to reach $10 \%$ degradation (the arbitrary point at which the lag phase is deemed to have finished in OECD RBTs ${ }^{1}$ ); half-life $\left(t_{50}\right)$, the time taken to reach $50 \%$ degradation; half-life excluding lag phase $\left(\mathrm{dt}_{50}\right.$; the time taken from the end of the lag phase to reach $50 \%$ degradation); and the maximum extent or level of degradation. Biodegradation data were also checked for their fit to common kinetic models (Supporting Information, Figures S1-7, Table $\left.S 1^{31}\right)$. Statistical analysis was performed using MINITAB (MINITAB 17, Coventry, U.K.).

Mass Balance Calculation. At the end of the experiment, recovery of the initially applied radioactivity was used to determine the fate and partitioning of the reference chemicals. Radioactivity, evolved as ${ }^{14} \mathrm{CO}_{2}$, was determined as previously described. Test vessel contents were filtered through Whatman grade 4 filter paper (Sigma-Aldrich, Poole, U.K.), and the filtrate was stored in preweighed 1L Nalgene bottles (ThermoFisher Scientific, Waltham, MA, U.S.A.), which were subsequently reweighed. Triplicate $5 \mathrm{~mL} \mathrm{NaOH}$ subsamples were mixed with a Gold Star scintillation cocktail (Meridian Biotechnologies Ltd., U.K.) and analyzed via LSC. Filter papers were combusted (A307 sample oxidizer: PerkinElmer, Waltham, MA, U.S.A.), and the product was made up to $20 \mathrm{~mL}$ with the Gold Star scintillation cocktail and analyzed via LSC. Orbo tubes (Sigma-Aldrich, Poole, U.K.) used to capture volatilized compounds were rinsed with $10 \mathrm{~mL}$ of ethanol, which was subsequently combined with the Gold Star scintillation cocktail and analyzed via LSC.

\section{RESULTS AND DISCUSSION}

BST Outcome. The erBST correctly assigned significantly more chemicals $(5 / 5)$ to their respective persistence categories $^{32-37}$ compared to the standard OECD RBT (2/5, ANOVA, $P<0.05)$. Use of the erBST was therefore accurate, resulting in no false negatives or false positive persistence assignations, whereas the OECD RBT falsely classified three chemicals (ANI, 4-FP, and 4-CA) as potentially persistent (i.e., false negatives) based on their current biodegradability classification $^{32-37}$ (Table 1). These chemicals would require further unnecessary higher tier testing under the current persistence assessment regime and may require bioaccumulation and/or toxicity testing depending on chemical properties, thereby incurring further testing costs and greater unnecessary regulatory oversight. The OECD RBTs were first implemented in the 1980s as conservative assessments to screen out chemicals that readily degraded in the environment. Therefore, both inherently and persistent chemicals should fail such tests. Even so, in our study two of the chemicals classified as readily biodegradable (ANI, 4-FP) also failed the RBT. This affirms evidence from previous studies about the unreliability, poor reproducibility, and variation in RBTs (e.g., ${ }^{18,23}$ ).

Importantly, the erBSTs were significantly more reproducible (ANOVA, $P<0.05$ ) (coefficient of variation, $\mathrm{CV}$, of replicate ${ }^{14} \mathrm{CO}_{2}$ measurements, $12 \%$ ) than the OECD RBT (CV 30\%), indicating that more consistent biodegradation test data was obtained at higher, more environmentally relevant inocula concentrations (Figure 1). This difference changed to CV $4 \%$ in the erBST and $34 \%$ in the RBT when only chemicals where
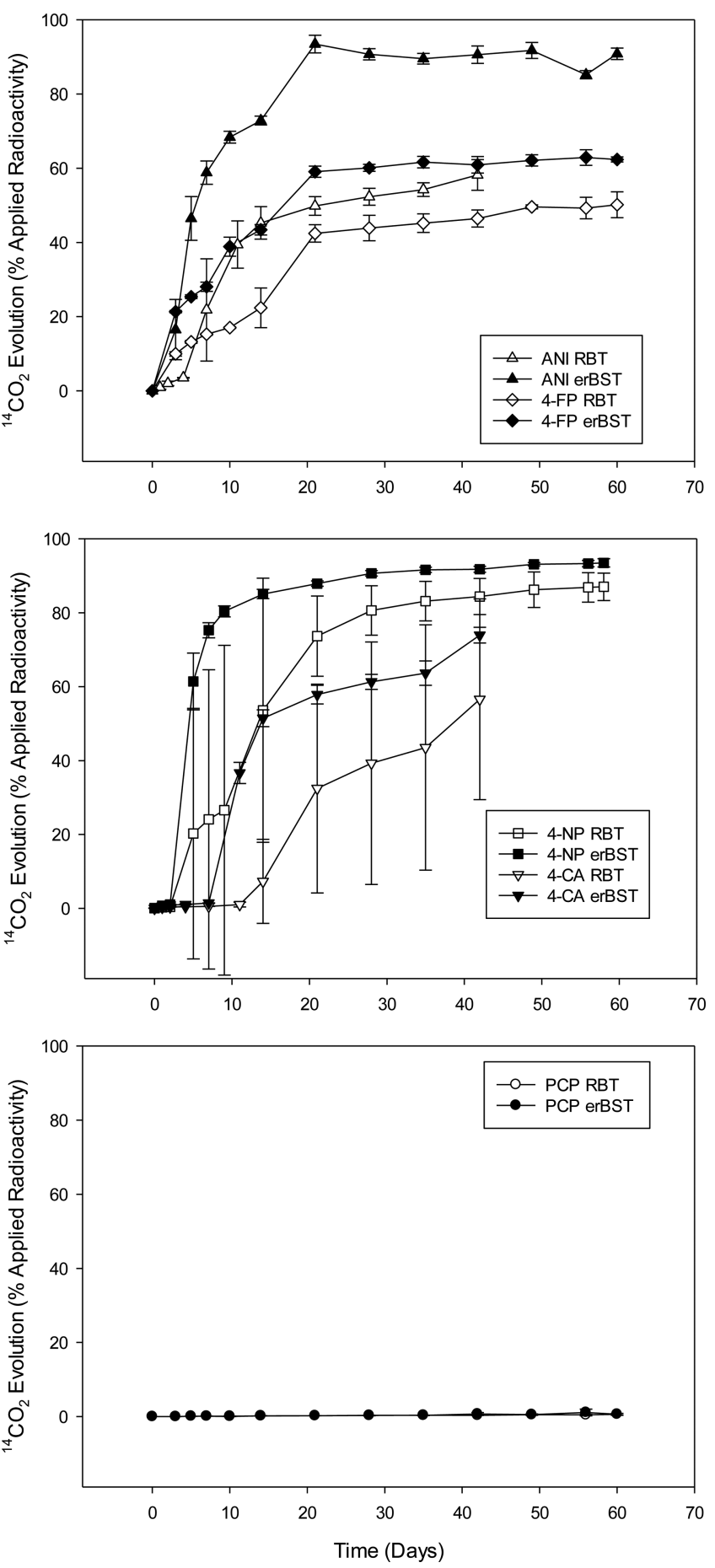

Figure 1. ${ }^{14} \mathrm{CO}_{2}$ evolution over time for the reference chemicals selected to validate the environmentally relevant biodegradation screening test. Data points are an average of triplicate biodegradation tests. Error bars are \pm 1 s.d. of triplicate ${ }^{14} \mathrm{CO}_{2}$ measurements. Plots indicate readily biodegradable, inherently/variably biodegradable and persistent compounds, based on ECHA Brief Profile classifications. ANI represents aniline; 4-FP, 4-fluorophenol; 4-NP, 4-nitrophenol; 4-CA, 4-chloroaniline and PCP, pentachlorophenol.

degradation was observed were considered. The erBST resulted in reduced inter-replicate variation, lag phase, and half-life duration, the time to reach $50 \%$ degradation after the lag phase $\left(\mathrm{dt}_{50}\right)$, and increased the maximum extent or level of degradation for all tested chemicals, where degradation was observed (Figures 1-3; Table 1). 


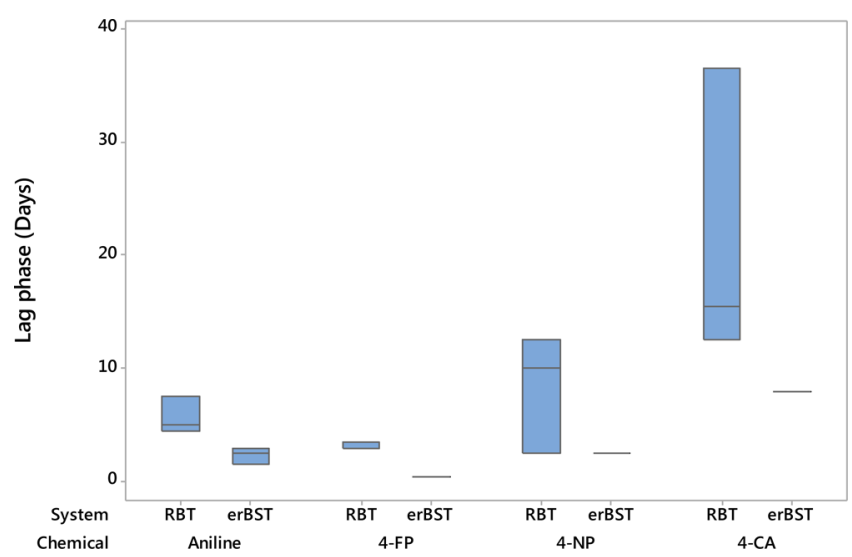

Figure 2. Boxplots indicating the range of lag phase durations (defined within OECD guidelines as the time taken to reach $10 \%$ degradation) for triplicate biodegradation tests, in erBSTs (erBST) and OECD RBTs (RBT) for a group of chemicals with varying environmental persistence. Higher inoculum concentrations give more consistent values with less variability between replicates.

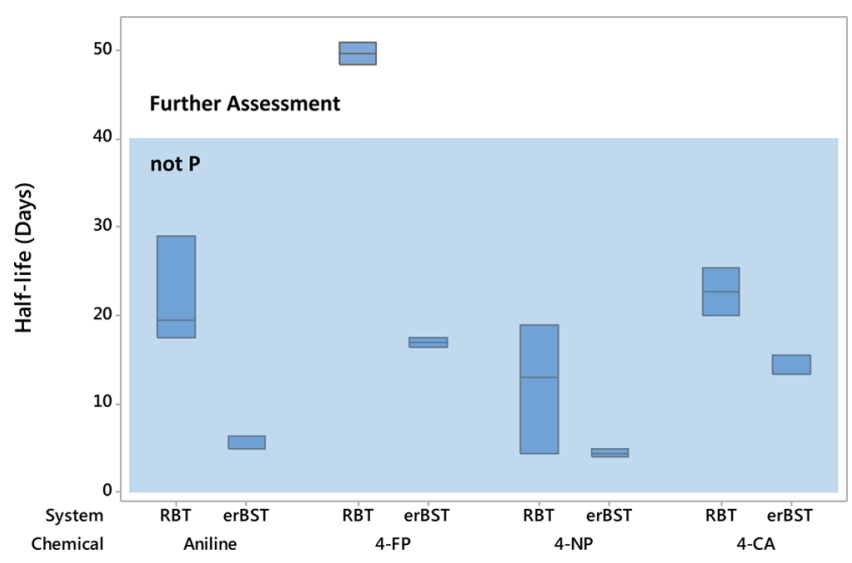

Figure 3. Boxplots indicating the range of half-life durations for triplicate biodegradation tests, in erBSTs and OECD RBTs for a group of chemicals with varying environmental persistence. Half-life is less than 20 days for all replicates of all readily and inherently/variably biodegradable compounds in erBSTs. Half-lives have been determined graphically using measured values. Higher inoculum concentrations give more consistent values with less variability between replicates. The shaded area indicates the erBST requirements for a compound to be classified as not persistent (not P).

Although half-lives were reduced in the erBST compared to the OECD RBT, regression analysis generally showed that data did not fit zero-order, first-order, logistic, or logarithmic growth kinetics (Supporting Information, Figures S1-3). Where sufficient degradation occurred to make a meaningful analysis, the models did not accurately follow the shape of the biodegradation, despite some $R^{2}$ values being suggestive of a good fit (Supporting Information, Table S1). This suggests that biodegradation most likely followed Monod-like kinetics even though growth of degraders could be proportionally insignificant due to the high starting biomass concentrations ${ }^{31}$ (Supporting Information, Figures S1-S7). More extensive modeling, and a more rigorous sampling regimen to include biomass concentration at each sample point, would be required to accurately describe the kinetics. The erBST may therefore be considered a conservative test based on the presence and activity of specific degraders.
The erBST correctly assigned all chemicals to their potential persistence category. ${ }^{32-37}$ The correct assignment of the positive reference compounds (ANI and 4-FP) and negative reference compound (PCP) suggest the test is neither overly protective nor overly powerful, essential characteristics of a regulatory biodegradation test. The inherently/variably biodegradable group of compounds (4-NP and 4-CA) were reproducibly characterized as not persistent by the erBST, indicating that the test possesses the ability to distinguish between those chemicals which should be screened out as nonpersistent and those which should be subject to further assessment. Values of $t_{\mathrm{L}}, t_{50}$, and extent of degradation were similar to those reported in the literature (Table 1$) ; t_{\mathrm{L}}$ and $t_{50}$ were consistently shorter and the extent of degradation consistently higher in the erBST than the RBT, where biodegradation was observed. Most importantly, the variation notoriously associated with OECD RBTs, reported in the literature and observed under RBT conditions in the present study, was reduced in the erBST which provided more consistent biodegradation test data with respect to replicate ${ }^{14} \mathrm{CO}_{2}$ measurements and the other degradation descriptors studied (Table 1). The $t_{\mathrm{L}}$ for 4-CA in the OECD RBT extended beyond 20 days (Figure 1; Table 1), and much of the degradation would have been missed had the study ended after the standard OECD RBT duration of 28 days. Although in these studies degradation in the erBST proceeded sufficiently rapidly that an extended test duration was not required, the potential for long lag phases when investigating biodegradable compounds, such as 4-CA, highlights the importance of increasing the test duration and establishing pass criteria which take into account the time required for degrading communities to establish and acclimate. This will be a key concern for chemicals with bioavailability issues, e.g., poorly water-soluble substances.

The pass threshold for an OECD RBT is currently defined as a $60 \%$ reduction in theoretical oxygen demand (ThOD) or theoretical $\mathrm{CO}_{2}$ evolution $\left(\mathrm{ThCO}_{2}\right)$, or a $70 \%$ reduction in DOC, within the 10-day window of a 28 day test. ${ }^{1}$ The lower threshold for respirometric methods acknowledges the incorporation of carbon from the test chemical into biomass. It has been previously argued that $60 \%$ is an unnecessarily high threshold for ThOD and $\mathrm{ThCO}_{2}{ }^{6,15,38}$ and that lowering to $50 \%$ would improve the consistency of reported pass/fail outcomes and therefore classification. ${ }^{13}$ There is evidence that as low as $40 \%$ ThOD may be equivalent to $80-100 \%$ DOC removal. ${ }^{6}$ The readily biodegradable positive reference compound used in this study (ANI) did not reach $60 \%$ degradation in the OECD RBT; however, it exceeded 50\% within 28 days, similar to previous studies which recommend that the respirometric pass limit should be lowered to $50 \%$ to improve classification..$^{13,15,38,39}$ Our radioactive mass balance indicated that a considerable amount of the carbon was incorporated into the biomass (1-11\% depending on chemical, Figure 4).

Mass balance data showed an average recovery of $93 \%$ of the initially applied radioactivity, ranging from $85.2 \%$ to $99.8 \%$ recovery, depending on the chemical and test system (Figure 4). Variation between replicates for mass balance recovery was typically low, with the exception of 4-CA in the OECD RBT, where two replicates degraded 4-CA in excess of $70 \%$, with the third replicate reporting $25 \%$ by study end. A considerable fraction of the remaining radioactivity for the third replicate was recovered in the filtrate, suggesting this replicate may have been in an extended lag phase (Figure 4). The ability to account for the majority of the applied radioactivity allowed us to determine the fate and partitioning of the compound during the 


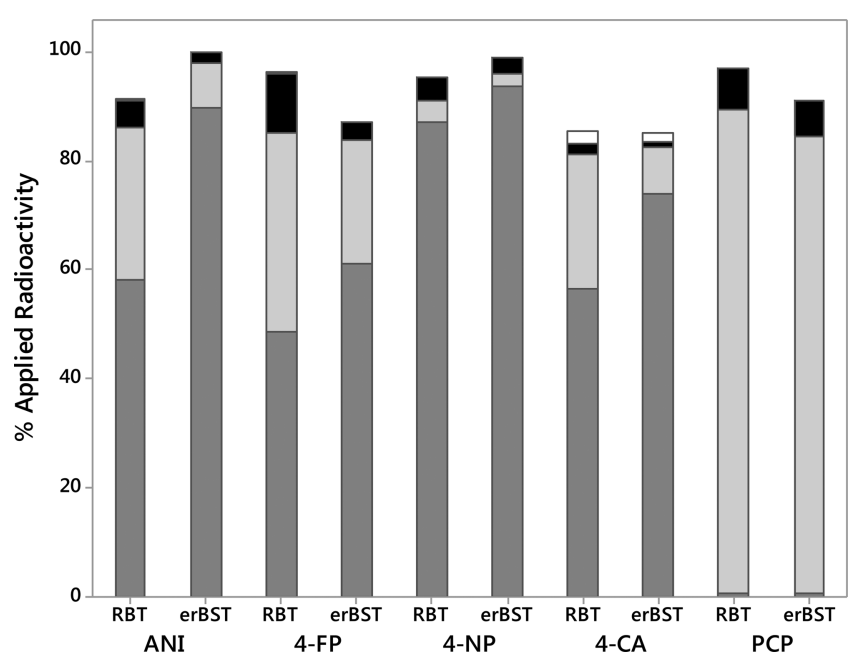

Figure 4. Mass balance at study end (ranging from day 42 to day 60 depending on study, see Figure 1) indicating recovery of initially applied radioactivity as evolved ${ }^{14} \mathrm{CO}_{2}$ (dark gray), in the aqueous phase following filtration (light gray), recovered from solids following combustion (black), and volatile organics (recovered from Orbo 32 and Orbo 91 tubes following methanol rinse) (white).

experiment to ${ }^{14} \mathrm{CO}_{2}$, its incorporation within the biomass, and/or its persistence in the aqueous phase. It ensured that our observations were not confounded by other potential losses (e.g., through volatility, sorption to glassware, issues with trapping compounds, or analytical sensitivity). It is important to note that the fraction remaining in the biomass was considered to be the ${ }^{14} \mathrm{C}$ from the test chemical incorporated into new biomass, rather than degradation products. We would recommend that validation or ring test biodegradation studies should use radioisotopes to accurately determine the fate of compounds and account for any losses. However, this should not limit the method from being conducted for routine use with other analytical approaches (e.g., $\mathrm{BOD}, \mathrm{CO}_{2}$, specific analysis) validated against the radioisotope approach. The increased inoculum concentration in the erBST compared to the RBT may lead to increased background respiration, although our preliminary research has suggested background levels may be sufficiently low for some RBT systems to operate, including the $301 \mathrm{~F}^{1}$ (data not shown). This will depend upon several factors, such as the analyte and the substrate concentration, which will result in some analytical methods being more suitable than others. Specific compound and transformation product analysis may be required to delineate the effect of background respiration in some scenarios. There are measures used within existing screening studies ${ }^{1,40}$ which could be adopted to reduce background respiration, for example, washing and slightly aging the inoculum. However, these methods may lead to undesirable decreases in inoculum diversity. ${ }^{18}$ The next step in the validation of the erBST would be to conduct a ring test across multiple laboratories, where background respiration could be assessed more thoroughly.

Our study adheres to many of the relevant criteria described within the OECD guidelines for the validation and acceptance of new or updated tests for hazard assessment and conforms to the relevant "Solna principles", which preceded them. ${ }^{41}$ The 8 Solna principles include: ensuring a clear scientific need and regulatory purpose for a new test; determination of intratest variability, repeatability, and reproducibility; use of a series of reference chemicals to determine method performance; and collection of data in accordance with the OECD principles of GLP. ${ }^{41}$ The following additional important criteria were highlighted in the OECD guidelines: the method provides data that adequately predict the end-point of interest and demonstrate a link between the new test and an existing test method; the method generates data that are preferably better than those obtained using existing methods; there are adequate testing data for representative chemicals; the test should be robust and transferrable and allow for standardization; the test is costeffective; and consideration should be given to animal welfare considerations including the $3 \mathrm{Rs}$. The main criteria that the current study failed to address was a comparison of the method across different laboratories, which would be required as the next step in validating the erBST, although it deviates little from the OECD 301B RBT that was part of a previous ring-test ${ }^{42}$ and which shows considerably more intrareplicate variability.

Regulatory Implications and Interpretation of Pass Criteria. Our data demonstrate that OECD RBTs are unreliable in both screening for, and making definitive classifications in, persistence assessments. In contrast, the erBST provided a robust prioritization on persistence, thereby providing the first validation that increased inocula concentrations, and extended test duration can improve the reliability of BSTs as previous studies have suggested. ${ }^{17,18,22}$ We suggest that the erBST can act as a single screening test that can identify and distinguish readily and inherently biodegradable chemicals from persistent chemicals. Although the utility of the test within $\mathrm{REACH}$ is restricted to $\mathrm{P}$ assessments, ${ }^{27}$ the test appears to be relevant and reliable enough to be used in exposure assessment and classification and labeling as evidence of rapid degradation, if the half-life is less than 16 days. ${ }^{43}$ The erBST includes an increased test duration and an inoculum concentration which enters the lower range of inherent biodegradability tests. ${ }^{44}$ Due to the robust prioritization on persistence offered, the erBST could end up being conducted more routinely than inherent tests, if the former were to prove reliable in a ring-test and receive regulatory acceptance. The goal of a robust erBST is to provide more reliable characterizations on persistence, thereby reducing the number of higher-tier assessments conducted, including inherent tests. However, its intended use would allow it to complement rather than replace inherent tests, which are slightly less stringent than erBSTs. For example, where there is a lack of observed degradation in an erBST but evidence, from the literature or chemical structure, that degradation may occur with acclimation and/or a higher inoculum concentration, it may be appropriate to perform an inherent test.

Regulatory guidance would be required to interpret and define pass threshold criteria for such a test. This may require a combination of half-life data, the extent of degradation, and/or degradation kinetics. We would recommend that the test should be run until plateau (for three determinations ${ }^{1}$ ) for a 60-day duration to encompass relevant half-life criteria for persistence stipulated under REACH. It should also be noted that even existing criteria for persistence are arbitrary and have no known scientific basis but are rather used as benchmarks against which chemicals can be compared. Pass criteria should therefore be defined for both positive and negative reference compounds. Time to reach $50 \%$ degradation has been recommended for persistence assessment in REACH guidance, ${ }^{3}$ with chemicals being classified as nonpersistent if they exhibit 50\% degradation in less than 40 days in freshwater systems and 60 days in marine systems. The OECD RBT guidelines acknowledge that respirometric methods will underestimate primary 
degradation (upon which persistence should be based), due to the incorporation of carbon from the test chemical into biomass. ${ }^{1}$ As stated previously others have advocated lowering the pass limit to $50 \%$ to improve the reliability of classifications, ${ }^{13,15,38,39}$ which is supported by data on aniline (ANI) degradation in this study. A lower threshold limit would be required for negative reference compounds, which would not be expected to pass a BST but may, under optimum conditions, exhibit some degradation in the environment. These criteria should only apply to the testing of single substances and would not be applicable to mixtures or substances of unknown or variable composition, complex reaction products, or biological materials (UVCBs). Where there is evidence of biodegradation occurring beyond 60 days, it may be prudent to extend the test in order to note the half-life from the end of lag phase $\left(\mathrm{dt}_{50}\right.$; typically judged as $10 \%$ degradation in OECD RBTs $\left.{ }^{1}\right) \cdot \mathrm{dt}_{50}$ values of less than 40 days in freshwater tests and less than 60 days in marine tests may be indicative that a chemical is not persistent.

Future Outlook. Increasing inocula toward more environmentally relevant concentrations that have more bacterial species and metabolisms representative of the environment allows accurate prediction of the relative persistence of chemicals when benchmarked against reference chemicals. Indeed, it also allows confident classification of those chemicals that can rapidly degrade in the environment. However, biodegradation testing is still stuck in the paradigm of one-off binary pass/fail tests to arbitrary threshold criteria that ignore the considerable variation between chemicals and between different inocula. Thus, the discipline of risk assessment, which has the definition of likelihood of doing harm at its very heart, makes no use of the variability and probabilities that could be harnessed from such biodegradation tests toward expressing likelihoods of degradation being observed. ${ }^{5,18}$ This has led to conservative tests with a high rate of false negatives and conflicting outcomes which inevitably lead to further costly biodegradation tests and unnecessary bioaccumulation and toxicity studies, which contravenes the 3 Rs principle. RBTs are rarely conducted with high replication due to the logistics and relatively high expense of doing so. However, there have been recent attempts to reinvent and reimagine biodegradation tests. ${ }^{5,18,45}$ These have followed the lead of new bioaccumulation and toxicity tests, whereby advances in miniaturization, throughput, and analyzing chemical degradation or mineralization could help to speed up, reduce the cost, and improve the basis of risk assessment based on probability of biodegradation. Inexpensive repeatable biodegradation tests may also allow more opportunities to better understand how microbial communities adapt and acclimate to new chemicals in the environment as previous studies have shown. ${ }^{25}$

\section{ASSOCIATED CONTENT}

\section{S Supporting Information}

The Supporting Information is available free of charge on the ACS Publications website at DOI: 10.1021/acs.est.6b05717.

Summary of degradation kinetics analysis. Plots detailing zero order, first order, logistical, and Monod no-growth curves fitted using linear and nonlinear regression. Summary tables of linear and nonlinear regression fits (PDF)

\section{AUTHOR INFORMATION}

\section{Corresponding Author}

*(T.J.M.) E-mail: timothy.martin@ncl.ac.uk. ORCID

Timothy J. Martin: 0000-0002-6555-9986

Present Address

§(A.B.) Ramboll Environ, Aston Court, Pynes Hill, Exeter, EX2 5AZ, United Kingdom.

\section{Author Contributions}

All authors have given approval to the final version of the manuscript.

\section{Notes}

The authors declare no competing financial interest.

\section{ACKNOWLEDGMENTS}

This work was funded by the European Chemical Industry Council (Cefic) Long-range Initiative (LRi; project ECO11) with in-kind support from AstraZeneca. R.J.D. would like to acknowledge funding from the Engineering and Physical Sciences Research Council (EPSRC) Challenging Engineering award (EP/I025782/1) and Research Council UK (RCUK) Academic Fellowship (EP500501/1). T.J.M. was funded through a BBSRC Industrial CASE award with AstraZeneca (BB/H530897/1). This work represents an AstraZeneca contribution to the Innovative Medicines Initiative (IMI) under grant agreement no. 115735-iPiE: Intelligent led assessment of Pharmaceuticals in the Environment. The authors would like to acknowledge the useful advice and discussions of the CEFIC LRi ECO11 research liaison team.

\section{ABBREVIATIONS}

3R's replacement, reduction, and refinement framework

4-CA 4-chloroaniline

4-FP 4-fluorophenol

4-NP 4-nitrophenol

ANI aniline

AS activated sludge

BOD biochemical oxygen demand

BST biodegradation screening test

CV coefficient of variation

DAPI 4',6-diamidino-2-phenylindole

DOC dissolved organic carbon

$\mathrm{dt}_{50}$ time to reach $50 \%$ degradation from the end of the lag phase

ECHA European Chemicals Agency

erBST environmentally relevant biodegradation screening test

GLP good laboratory practice

LSC liquid scintillation counting

OECD Organisation for Economic Co-operation and Development

PBT persistent, bioaccumulative, toxic

PCP pentachlorophenol

RBT ready biodegradability test

$\mathrm{REACH}$ registration, evaluation, authorization, and restriction of chemicals

$t_{50} \quad$ time to reach $50 \%$ degradation

$\mathrm{ThCO}_{2}$ theoretical carbon dioxide production

ThOD theoretical oxygen demand

$t_{\mathrm{L}} \quad$ lag phase 


\section{REFERENCES}

(1) OECD. OECD Guideline for testing of chemicals: 301 Ready Biodegradability; 1992.

(2) Kowalczyk, A.; Martin, T. J.; Price, O. R.; Snape, J. R.; van Egmond, R. A.; Finnegan, C. J.; Schäfer, H.; Davenport, R. J.; Bending, G. D. Refinement of biodegradation tests methodologies and the proposed utility of new microbial ecology techniques. Ecotoxicol. Environ. Saf. 2015, 111 (0), 9-22.

(3) ECHA. Guidance on information requirements and chemical safety assessment. Chapter R.7b: Endpoint specific guidance; European Chemicals Agency: 2012; p 238.

(4) ECETOC. Assessing environmental persistence. Workshop Report No. 24; 2012.

(5) Thouand, G.; Durand, M. J.; Maul, A.; Gancet, C.; Blok, H. New Concepts in the Evaluation of Biodegradation/Persistence of Chemical Substances using a Microbial Inoculum. Front. Microbiol. 2011, 2, 164.

(6) Painter, H. A. Detailed review paper on biodegradability testing: Environment monograph No. 98; Organisation for Economic Cooperation and Development: Paris, 1995.

(7) Boethling, R.; Fenner, K.; Howard, P.; Klečka, G.; Madsen, T.; Snape, J. R.; Whelan, M. J. Environmental Persistence of Organic Pollutants: Guidance for Development and Review of POP Risk Profiles. Integr. Environ. Assess. Manage. 2009, 5 (4), 539-556.

(8) ECETOC. Workshop on biodegradation and persistence. Workshop Report No. 10; 2007.

(9) Shrestha, P.; Junker, T.; Fenner, K.; Hahn, S.; Honti, M.; Bakkour, R.; Diaz, C.; Hennecke, D. Simulation Studies to Explore Biodegradation in Water-Sediment Systems: From OECD 308 to OECD 309. Environ. Sci. Technol. 2016, 50 (13), 6856-6864.

(10) Gerike, P.; Fischer, W. K. Correlation study of biodegradability determinations with various chemicals in various tests. Ecotoxicol. Environ. Saf. 1979, 3 (2), 159-173.

(11) Gerike, P.; Fischer, W. K. A correlation study of biodegradability determinations with various chemicals in various tests 2 . Additional results and conclusions. Ecotoxicol. Environ. Saf. 1981, 5 (1), 45-55.

(12) Zgajnar Gotvajn, A.; Zagorc-Koncan, J. Laboratory simulation of biodegradation of chemicals in surface waters: Closed bottle and respirometric test. Chemosphere 1999, 38 (6), 1339-1346.

(13) Boethling, R. S.; Lynch, D. G. Biodegradation of US premanufacture notice chemicals in OECD tests. Chemosphere 2007, 66 (4), 715-722.

(14) Nyholm, N.; Lindgaard-Jørgensen, P.; Hansen, N. Biodegradation of 4-nitrophenol in standardized aquatic degradation tests. Ecotoxicol. Environ. Saf. 1984, 8 (5), 451-470.

(15) Boethling, R.; Lynch, D. G.; Thom, G. C. Predicting ready biodegradability of premanufacture notice chemicals. Environ. Toxicol. Chem. 2003, 22 (4), 837-844.

(16) Reuschenbach, P.; Pagga, U.; Strotmann, U. A critical comparison of respirometric biodegradation tests based on OECD 301 and related test methods. Water Res. 2003, 37 (7), 1571-1582.

(17) Thouand, G.; Friant, P.; Bois, F.; Cartier, A.; Maul, A.; Block, J. C. Bacterial Inoculum Density and Probability of para-Nitrophenol Biodegradability Test Response. Ecotoxicol. Environ. Saf. 1995, 30 (3), 274-282.

(18) Goodhead, A.; Head, I.; Snape, J.; Davenport, R. Standard inocula preparations reduce the bacterial diversity and reliability of regulatory biodegradation tests. Environ. Sci. Pollut. Res. 2014, 21, 9511.

(19) Nyholm, N.; Kristensen, P. Screening methods for assessment of biodegradability of chemicals in seawater-Results from a ring test. Ecotoxicol. Environ. Saf. 1992, 23 (2), 161-172.

(20) Thouand, G.; Block, J. C. The use of precultured inocula for biodegradability tests. Environ. Technol. 1993, 14 (7), 601-614.

(21) Vázquez-Rodríguez, G. A.; Garabétian, F.; Rols, J.-L. Inocula from activated sludge for ready biodegradability testing: Homogenization by preconditioning. Chemosphere 2007, 68 (8), 1447-1454.

(22) Ingerslev, F.; Torang, L.; Nyholm, N. Importance of the test volume on the lag phase in biodegradation studies. Environ. Toxicol. Chem. 2000, 19 (10), 2443-2447.
(23) Thouand, G.; Capdeville, B.; Block, J. C. Preadapted Inocula for Limiting the Risk of Errors in Biodegradability Tests. Ecotoxicol. Environ. Saf. 1996, 33 (3), 261-267.

(24) Toräng, L.; Nyholm, N. Biodegradation rates in adapted surface water can be assessed following a preadaptation period with semicontinuous operation. Chemosphere 2005, 61 (1), 1-10.

(25) Itrich, N. R.; McDonough, K. M.; van Ginkel, C. G.; Bisinger, E. C.; LePage, J. N.; Schaefer, E. C.; Menzies, J. Z.; Casteel, K. D.; Federle, T. W. Widespread Microbial Adaptation to 1-Glutamate-N,Ndiacetate (L-GLDA) Following Its Market Introduction in a Consumer Cleaning Product. Environ. Sci. Technol. 2015, 49 (22), 13314-13321.

(26) ECETOC. Persistence of chemicals in the environment. Workshop Report No. 90; 2003.

(27) ECHA. Guidance on information requirements and chemical safety assessment. Chapter R.11: PBT assessment, Version 2.0; 2014.

(28) OECD eChem portal: The global portal to information on chemical substances. http://www.echemportal.org/echemportal/ index.action; April 2, 2013.

(29) Comber, M.; Holt, M. ECO 12: Developing a set of reference chemicals for use in biodegradability tests for assessing the persistency of chemicals. Report No: MCC/007; 2010.

(30) Davenport, R. J.; Curtis, T. P., Quantitative fluorescence in situ hybridisation (FISH): statistical methods for valid cell counting. In Molecular microbial ecology manual; Kowalchuk, G. A., de Bruijn, F. J., Head, I. M., Akkermans, A. D., van Elsas, J. D., Eds.; Springer-Verlag: New York, 2004; pp 1487-1516.

(31) Simkins, S.; Alexander, M. Models for mineralization kinetics with the variables of substrate concentration and population density. Appl. Environ. Microbiol. 1984, 47 (6), 1299-1306.

(32) ECHA Brief Profile - 4-fluorophenol. https://echa.europa.eu/ brief-profile/-/briefprofile/100.006.124 (accessed December 20, 2016).

(33) ECHA Brief Profile - aniline. https://echa.europa.eu/briefprofile/-/briefprofile/100.000.491 (accessed December 20, 2016).

(34) ECHA Brief Profile - 4-nitrophenol. https://echa.europa.eu/ brief-profile/-/briefprofile/100.002.556 (accessed December 20, 2016).

(35) ECHA Brief Profile - 4-chloroaniline. https://echa.europa.eu/ brief-profile/-/briefprofile/100.003.093 (accessed December 20, 2016).

(36) ECHA Substance Information - Pentachlorophenol. https:// echa.europa.eu/substance-information/-/substanceinfo/100.001.617 (accessed December 20, 2016).

(37) SYKE The Finnish Environment Institute (SYKE): Data bank of environmental chemicals - Pentachlorophenol. http://wwwp. ymparisto.fi/scripts/Kemrek/Kemrek_uk.asp? Method= MAKECHEMdetailsform\&txtChemId=245 (accessed December 20, 2016).

(38) Painter, H. A.; King, E. F. A respirometric method for the assessment of ready biodegradability: Results of a ring test. Ecotoxicol. Environ. Saf. 1985, 9 (1), 6-16.

(39) Junker, T.; Paatzsch, C.; Knacker, T. A water-sediment screening tool for measuring biodegradation of organic chemicals. Sci. Total Environ. 2010, 408 (18), 3803-3810.

(40) OECD. OECD guideline for testing of chemicals: OECD 306 Biodegradability in seawater; 1992.

(41) OECD. OECD Environment, Health and Safety Publications Series on Testing and Assessment No 34. Guidance document on the validation and international acceptance of new or updated test methods for hazard assessment; 2005.

(42) OECD. OECD Ring-test of methods for determining ready biodegradability: Chairman's report (M. Hashimoto, MITI) and final report (M. Kitano and M. Takatsuki, CITI). Paris; 1988.

(43) OECD. Harmonised integrated classification system for human health and environmental hazards of chemical substances and mixtures. OECD series on testing and assessment. No 33; 2001.

(44) OECD. OECD guideline for testing of chemicals 302C: Modified MITI test (II); 1981. 
(45) Cregut, M.; Jouanneau, S.; Brillet, F.; Durand, M. J.; Sweetlove, C.; Cheneble, J. C.; L'Haridon, J.; Thouand, G. High throughput and miniaturised systems for biodegradability assessments. Environ. Sci. Pollut. Res. 2014, 21 (16), 9545-9552.

(46) Ahtiainen, J.; Aalto, M.; Pessala, P. Biodegradation of chemicals in a standardized test and in environmental conditions. Chemosphere 2003, 51 (6), 529-537.

(47) Ingerslev, F.; Nyholm, N. Shake-flask test for determination of biodegradation rates of C-14-labeled chemicals at low concentrations in surface water systems. Ecotoxicol. Environ. Saf. 2000, 45 (3), 274283.

(48) Chaojie, Z.; Qi, Z.; Ling, C.; Yuan, Y.; Hui, Y. Degradation of mono-fluorophenols by an acclimated activated sludge. Biodegradation 2007, 18 (1), 51-61.

(49) ECHA 4-fluorophenol registration dossier. https://echa.europa.eu/ registration-dossier/-/registered-dossier/12102/1 (accessed December 14, 2015).

(50) Goodhead, A. K. Towards rational risk assessment: Improving biodegradation tests through an understanding of microbial diversity. $\mathrm{PhD}$ dissertation, Newcastle University, U.K., 2009.

(51) Lapertot, M. E.; Pulgarin, C. Biodegradability assessment of several priority hazardous substances: Choice, application and relevance regarding toxicity and bacterial activity. Chemosphere 2006, 65 (4), 682-690.

(52) Ingerslev, F.; Baun, A.; Nyholm, N. Aquatic biodegradation behavior of pentachlorophenol assessed through a battery of shake flask die-away tests. Environ. Toxicol. Chem. 1998, 17 (9), 1712-1719. 